\title{
Clinicopathological features and prognosis of
}

\section{AFP-producing colorectal cancer: a single-center analysis of 20 cases}

This article was published in the following Dove Press journal:

Cancer Management and Research

\author{
Fei $\operatorname{Ren}^{1,2}$ \\ Weiwei Weng ${ }^{1,2}$ \\ Qiongyan Zhang ${ }^{\text {I,2 }}$ \\ Cong Tan ${ }^{1,2}$ \\ Midie $X u^{1,2}$ \\ Meng Zhang ${ }^{1,2}$ \\ Lei Wang ${ }^{1,2}$ \\ Weiqi Sheng ${ }^{1,2}$ \\ Shujuan $\mathrm{Ni}^{1,2, *}$ \\ Dan Huang ${ }^{1,2, *}$ \\ 'Department of Pathology, Fudan \\ University Shanghai Cancer Center, \\ Shanghai, People's Republic of China; \\ ${ }^{2}$ Department of Oncology, Shanghai \\ Medical College, Fudan University, \\ Shanghai, People's Republic of China
}

*These authors contributed equally to this work
Correspondence: Shujuan Ni; Dan Huang Fudan University Shanghai Cancer Center, 270 Dongan Road, Shanghai 200032,

People's Republic of China

Tel +862164175590

Email nsj616@126.com;

dianehuangfdcc@gmail.com
Background: High serum levels of alpha-fetoprotein (AFP) are observed in some gastrointestinal cancers. However, primary AFP-producing colorectal cancer (CRC) is extremely rare and causes confusion among clinicians. In this study, we analyzed the clinicopathological features and clinical outcomes of AFP-producing CRC and provide a brief view of this rare carcinoma. Patients and methods: Twenty patients with AFP-producing CRC were enrolled at the Fudan University Shanghai Cancer Center from 2012 to 2015. Clinical information, including serum AFP and CEA levels, and outcomes were collected. Tumors were divided into three histologic types: the common adenocarcinoma (COM) type, mucinous adenocarcinoma type and hepatoid type (HPT). Immunohistochemical (IHC) staining of GPC3, Hepa-1, SALL4 and Arg-1 was performed. Additionally, mutations of the KRAS, NRAS and BRAF genes were examined. Finally, another 40 stage-matched patients with traditional CRC were enrolled as controls for survival analysis.

Results: AFP-producing CRC was more likely to occur in males (60\%) and arose mainly from the ascending (40\%) and sigmoid (35\%) colon. In addition, the majority of patients with AFPproducing CRC had poor differentiation (50\%), advanced local invasion (80\%) and lymph node (LN) metastasis (60\%). Synchronous distant metastasis was commonly observed (35\%). Interestingly, serum AFP levels were closely associated with LN metastasis. Histopathologically, the COM type was the most common pattern. In IHC staining, the HPT pattern was the most distinct due to high positivity rates of GPC3, Hepa-1 and Arg-1. One patient had mismatch repair deficiency, and another had a KRAS mutation. Patients with AFP-producing CRC had worse progression-free and overall survival than patients with traditional CRC.

Conclusion: AFP-producing CRC has unique clinical and histopathological characteristics, showing an aggressive biological behavior and worse prognosis than traditional CRC.

Keywords: AFP-producing colorectal cancer, clinicopathological features, serum AFP, prognosis

\section{Introduction}

Alpha-fetoprotein (AFP) was initially found in a considerable concentration in human fetal sera. ${ }^{1}$ Serum AFP levels decrease after birth; thus, elevated AFP is abnormal in adults. AFP is usually elevated in patients with hepatocellular carcinomas or yolk sac tumors. ${ }^{2,3}$ Hence, testing AFP in serum is a useful method for screening and monitoring these patients. AFP-producing tumors have mainly been reported in organs originating from the foregut endoderm. ${ }^{4}$ The majority of AFP-producing cancers originate from the stomach, bile duct, and pancreas and show a high incidence of liver metastasis and a poor 
prognosis. ${ }^{5}$ However, AFP-producing colorectal cancer (CRC) is extremely rare. ${ }^{6-17}$ One reason for this might be that colorectal tissues originate from the hindgut endoderm, differing from stomach tissues, which develop from the foregut. Since the first report of AFP-producing CRC by Nakajima et al in 1985, only 14 cases of AFP-producing cancer ${ }^{6-17}$ and several reports of hepatoid adenocarcinoma ${ }^{18-25}$ in the colorectum have been sporadically published in English literature to date. However, extensive data regarding the clinicopathological profiles and prognosis of AFP-producing CRC remain limited, with most studies in the literature being case reports.

In this study, we aimed to summarize the clinicopathological features and survival outcomes of primary AFP-producing CRC. Further immunohistochemical (IHC) and molecular studies were performed to clarify this unique tumor type.

\section{Patients and methods}

\section{Patient selection}

We studied 20 patients with primary AFP-producing CRC who underwent surgical resection at Fudan University Shanghai Cancer Center (FUSCC) from 2012 to 2015. Another 40 stage-matched patients with traditional CRC (non-AFP-producing CRC) were also enrolled as a control group from 2012 to 2014. The control group has the similar background of age, gender, location and differentiation. Signed informed consent was obtained from all patients and the study was approved by the Clinical Research Ethics Committee of FUSCC. The inclusion criteria were as follows: the presence of primary sporadic tumors with definite diagnosis, elevated serum AFP $(>10.00 \mathrm{ng} / \mathrm{mL})$ in patients with AFP-producing CRC and normal serum AFP in patients with traditional CRC (non-AFP-producing CRC). The diagnosis was histopathologically confirmed by two pathologists, and histological findings were recorded. Clinical data, including age and gender, were obtained from medical records. All patients were prescribed a follow-up regimen based on the National Comprehensive Cancer Network guidelines of colon and rectal cancer, with physical, serum, imaging and colonoscopy examination timely. Cancer and vital status were recorded by clinical follow-up at our center.

\section{Serum assays for AFP and CEA}

Blood samples were obtained from all patients in the morning during the week before and the week after surgery. The blood sample was centrifuged at $4000 \mathrm{rpm}$ for 10 mins to separate the plasma from the blood cells. AFP and carcinoembryonic antigen (CEA) were assayed using a Cobas detection kit (Roche, Basel, $\mathrm{CH}$ ) according to the manufacturer's instructions. The cutoff values for serum AFP and CEA were 10.00 and $5.20 \mathrm{ng} / \mathrm{mL}$, respectively.

\section{IHC studies}

All tumors with unstained slides were available. IHC staining was performed using Ventana automation with the corresponding detection system (Ventana Medical Systems, Tucson, AZ, USA) and antibodies against GPC3 (clone 1G12, prediluted; Maixin, Fuzhou, Fujian, People'sRepublic of China), SALL4 (clone 6E3, 1:100; Biocare, Pacheco, CA, USA), Arg-1 (monoclonal, 1:6,000; Sigma-Aldrich, St. Louis, MO, USA), and Hepa-1 (clone OCH1E5, 1:100, Maixin).

Microsatellite instability was assessed using IHC staining for mismatch repair (MMR) proteins, including MLH1 (clone M1, prediluted; Ventana), PMS2 (clone A16-4, prediluted; Ventana), MSH2 (clone G2- 19-1129, prediluted; Ventana) and MSH6 (clone SP93, prediluted; Ventana). Loss of expression of any of the MMR proteins was considered when there was no corresponding IHC staining in the nuclei in tumor cells, whereas adjacent normal colonic mucosa and/ or stromal cells had nuclear staining by contrast.

\section{Molecular studies}

For DNA extraction, the QIAamp DNA mini kit (Qiagen, Manchester, UK) was used, employing the manufacturer's standard protocol. Primers for amplification and pyrosequencing were designed using Pyrosequencing Assay Design Software (Qiagen, Manchester, UK). All the patients were tested for mutations in KRAS exons 2, 3, 4, NRAS exons 2, 3, 4 and BRAF exon 15. Each sample was sequenced by an ABI 3500XL instrument using a BigDye Terminator v. 3.1 Cycle Sequencing kit (Applied Biosystems, Carlsbad, CA, USA). All samples were tested by bidirectional sequencing, and the positive samples were confirmed by two independent experiments.

\section{Statistical analysis}

SPSS 20.0 package (IBM, Chicago, IL, USA) and GraphPad Prism 7.0 software (GraphPad Software, Inc., La Jolla, CA, USA) were used for statistical analyses and scientific graphing, respectively. Comparisons between groups were determined by two-sided Mann-Whitney U tests or Fisher's exact test, as appropriate. Kaplan-Meier survival curves were used to compare survival rates. $P$-values $<0.05$ were considered statistically significant. 


\section{Results}

\section{Clinicopathological features}

The clinicopathological characteristics of the 20 AFPproducing CRC patients are summarized in Table 1. There were 12 males $(60 \%)$ and 8 females $(40 \%)$ (male to female ratio $=1.5: 1)$. The median age was 60 years, ranging from 33 to 80 years. Eight cases $(40 \%)$ arose from the ascending colon, 7 cases (35\%) arose from the sigmoid colon, 4 cases (20\%) arose from the rectum and only 1 case (5\%) arose from the descending colon. Ten cases $(50 \%)$ showed poor differentiation, 9 cases $(45 \%)$ showed moderate differentiation and only one case showed good differentiation.

Table I Clinicopathological characteristics of 20 patients with AFP-producing CRC

\begin{tabular}{|l|l|}
\hline Characteristics & N (\%) \\
\hline Age (years) & \\
$\leq 60$ & $10(50 \%)$ \\
$>60$ & $10(50 \%)$ \\
\hline Sex & \\
Male & $12(60 \%)$ \\
Female & $8(40 \%)$ \\
\hline Location & \\
Ascending & $8(40 \%)$ \\
Descending & $1(5 \%)$ \\
Sigmoid & $7(35 \%)$ \\
Rectum & $4(20 \%)$ \\
\hline Differentiation & \\
Poorly differentiated & $10(50 \%)$ \\
Moderately differentiated & $9(45 \%)$ \\
Well differentiated & $1(5 \%)$ \\
\hline Depth & \\
TI+T2 & $4(20 \%)$ \\
T3+T4 & $16(80 \%)$ \\
\hline LN metastasis & $7(35 \%)$ \\
Yes & $12(60 \%)$ \\
No & $8(40 \%)$ \\
\hline Distant metastasis & $13(65 \%)$ \\
Yes & \\
No & $7(5 \%)$ \\
\hline PTNM stage ${ }^{\text {II }}$ & \\
\hline
\end{tabular}

Notes: ${ }^{\text {a }}$ atients were staged in accordance with the 8th edition of the AJCC TNM classification.

Abbreviations: AJCC, American Joint Committee on Cancer; CRC, colorectal cancer; LN, lymph node.
With regard to the depth of tumor invasion, early local invasion (pT1+T2) was observed in 4 patients $(20 \%)$, and advanced local invasion (pT3+T4) was observed in 16 patients $(80 \%)$. Lymph node (LN) metastasis was observed in 12 patients $(60 \%)$. Synchronous distant metastasis was observed in 7 patients (35\%), including 6 with liver metastases and 1 patient with peritoneum metastasis. The clinicopathological characteristics of the control group are summarized in Table S1.

\section{Serum AFP levels}

All 20 patients had elevated preoperative serum AFP levels ranging from 12.61 to $181.4 \mathrm{ng} / \mathrm{mL}$ (median $24.16 \mathrm{ng} / \mathrm{mL}$ ). The level of serum AFP decreased significantly after the surgery, and the postoperative serum AFP levels ranged from 2.76 to 21.1 (median $6.2 \mathrm{ng} / \mathrm{mL}$ ) (Table 2). Eleven cases (55\%) had elevated preoperative serum CEA. We further analyzed the association between preoperative serum AFP and clinicopathological parameters. When the 20 samples were stratified based on LN metastasis status, serum AFP was further significantly higher in AFP-producing CRC patients with LN metastasis (Figure 1A). However, there was no significant difference between the AFP level and local invasion ( $\mathrm{T}$ stage) or between distant metastasis and TNM stage (Figure 1B-D).

\section{Pathological findings}

The morphology in our cases was classified into three patterns: the common adenocarcinoma type (COM), mucinous adenocarcinoma (MA) type and hepatoid type component (HPT). MA was observed in 4 cases $(20 \%)$, COM alone was observed in 10 cases $(50 \%)$, the mixed type $(\mathrm{COM}+\mathrm{HPT})$ was observed in 5 cases $(25 \%)$, and HPT alone was observed in only one case (5\%) (Table 3, Figure $2 \mathrm{~A}$ and $\mathrm{B}$ ). None of the cases showed an enteroblastic or yolk sac tumor pattern.

For IHC staining, 5 of 20 cases (25\%) showed variable GPC-3 expression, Hepa-1 was moderately to diffusely positive in 7 cases (35\%), and Arg-1 was positive in 5 cases $(25 \%)$ (Figure 2C-E). SALL4 was negative in all cases. Notably, the expression of GPC3, Hepa-1 and Arg-1 was more likely to be found in the HPT pattern, with positive rates of $66.7 \%, 83.3 \%$ and $66.7 \%$, respectively. Differences of the expression of these three markers between the HPT pattern and non-HPT pattern were statistically significant $(P<0.05)$ (Figure 3$)$.

Only one patient with the COM type exhibited MMR deficiency (complete loss of MLH1 and PMS2), and 
Table 2 Changes of serum level of AFP and CEA at the time of preoperation and postoperation

\begin{tabular}{|c|c|c|c|c|}
\hline Case & $\begin{array}{l}\text { Preoperative AFP level } \\
(\mathrm{ng} / \mathrm{mL})\end{array}$ & $\begin{array}{l}\text { Postoperative AFP level } \\
(\mathrm{ng} / \mathrm{mL})\end{array}$ & $\begin{array}{l}\text { Preoperative CEA level } \\
(\mathrm{ng} / \mathrm{mL})\end{array}$ & $\begin{array}{l}\text { Postoperative CEA level } \\
(\mathrm{ng} / \mathrm{mL})\end{array}$ \\
\hline I & 20.23 & 2.76 & 2.46 & 2.08 \\
\hline 2 & 181.4 & 21.1 & 82.32 & 12.7 \\
\hline 3 & 13.87 & 3.42 & I.II & 2.21 \\
\hline 4 & 29.58 & 8.52 & 6.34 & 31.63 \\
\hline 5 & 34.79 & 5.61 & 45.48 & 23.54 \\
\hline 6 & 57.9 & 3.77 & 13.39 & 3.32 \\
\hline 7 & 32.34 & 14.13 & 2.57 & 1.86 \\
\hline 8 & 16.04 & 10.35 & 8.28 & 8.85 \\
\hline 9 & 24.39 & 3.73 & 12.24 & 4.26 \\
\hline 10 & 16.81 & 5.51 & 5.28 & 3.93 \\
\hline II & 23.94 & 9.18 & 1.92 & 1.35 \\
\hline 12 & 23.76 & 3.9 & 37.65 & 43.75 \\
\hline 13 & 15.5 & 9.02 & 3.89 & 1.37 \\
\hline 14 & 15.11 & 6.79 & 2.55 & 2.42 \\
\hline 15 & 28.03 & 17.54 & 1.93 & 2.17 \\
\hline 16 & 46.3 & 3.17 & 2.61 & 3.38 \\
\hline 17 & 71.42 & $\mid 4.74$ & 4.4 & 3.91 \\
\hline 18 & $12.6 \mathrm{I}$ & 8.71 & 12.98 & 14.76 \\
\hline 19 & 30.01 & 3.34 & 63.98 & 94.36 \\
\hline 20 & 18.5 & 3.22 & 7.37 & 6.7 \\
\hline
\end{tabular}

Abbreviations: AFP, alpha-fetoprotein; CEA, carcinoembryonic antigen.

A

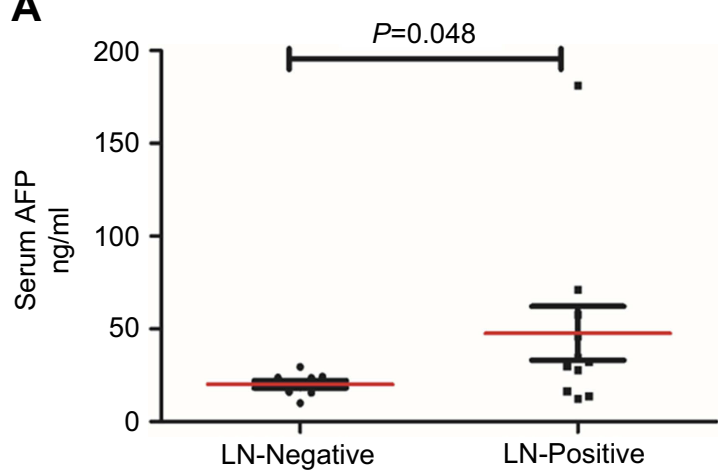

C

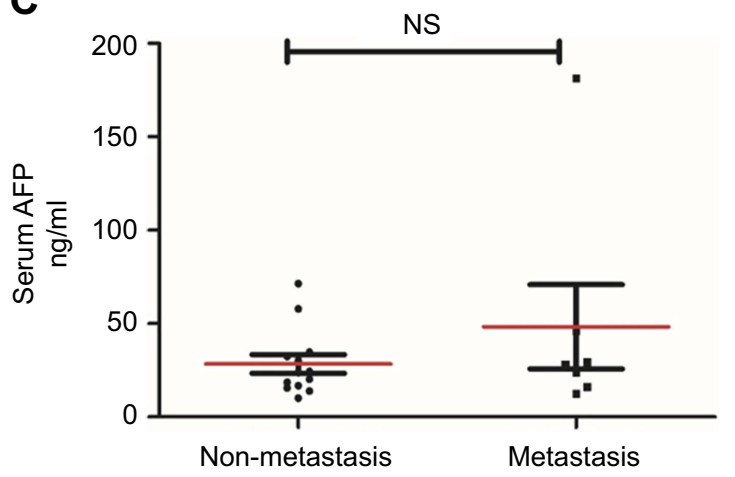

B

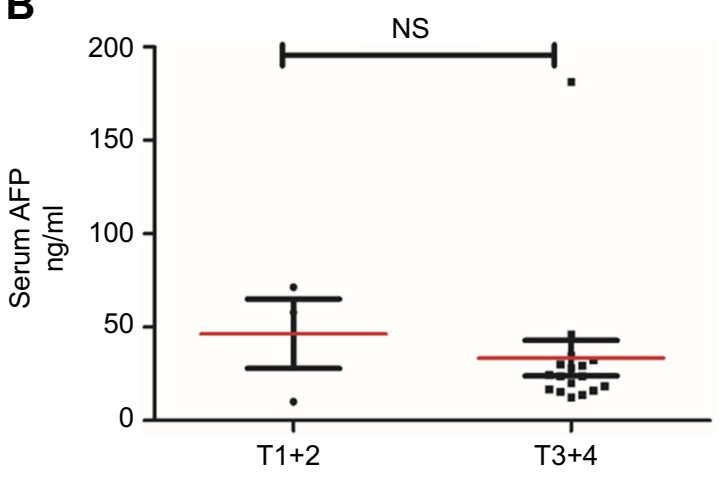

D

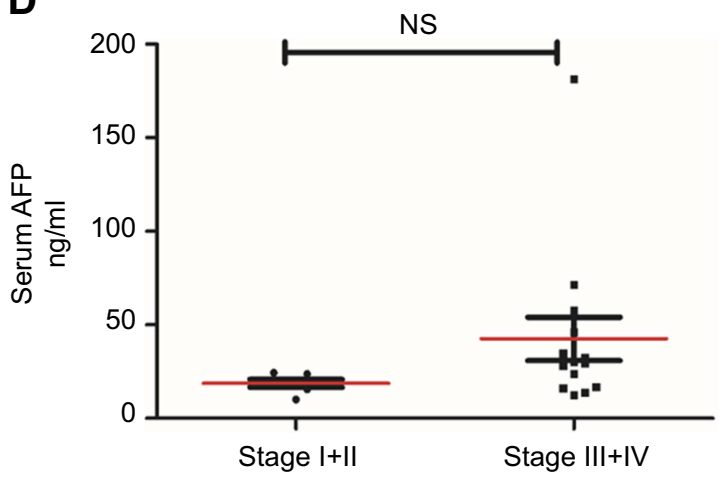

Figure I Statistical analysis of the serum AFP between different clinicopathological parameters. (A) The serum AFP levels in patients with LN metastasis were significantly higher than those in patients without LN metastasis. There were no significant differences between the AFP level and local invasion (T stage) (B), distance metastasis (C), or TNM stage (D). Abbreviations: LN, lymph node; NS, not significant. 
Table 3 Immunohistochemical and molecular findings in AFP-producing CRC

\begin{tabular}{|l|l|l|l|l|l|l|l|}
\hline Case & Histologic pattern & GPC-3 & Hepa-I & SALL4 & Arg-I & MMR & KRAS/NRAS/BRAF \\
\hline 1 & MA & - & - & - & - & - & - \\
2 & COM+HPT & + & + & - & - & - & - \\
3 & HPT & + & + & - & + & - & - \\
4 & COM+HPT & - & + & - & - & - & - \\
5 & COM & - & + & - & - & dMMR & - \\
6 & COM & - & - & - & - & - & - \\
7 & MA & - & - & - & - & - & - \\
8 & COM & + & - & - & + & - & - \\
9 & COM & - & + & - & - & - & - \\
10 & MA & - & - & - & - & - & - \\
11 & COM & - & - & - & - & - & - \\
12 & COM+HPT & - & + & - & + & - & - \\
13 & COM+HPT & + & + & - & + & - & - \\
14 & COM & - & - & - & - & - & - \\
15 & COM & - & - & - & - & - & - \\
16 & COM & - & - & - & - & - & - \\
17 & COM+HPT & + & - & - & + & - & - \\
18 & MA & - & - & - & - & - & KRAS GI46A \\
19 & COM & - & - & - & - & - & - \\
20 & COM & - & - & - & - & - & - \\
\hline
\end{tabular}

Abbreviations: COM, common adenocarcinoma type; CRC, colorectal cancer; dMMR, mismatch repair deficient; HPT, hepatoid type; MA, mucinous adenocarcinoma; MMR, mismatch repair.

another patient with MA type harbored a KRAS mutation in exon 4 (G146A) (Table 3).

\section{Follow-up}

Survival data of the 20 patients were eventually selected for analysis. The follow-up period ranged from 10 to 64 months (mean, 36 months; median, 35 months). Nine patients experienced disease progression, and 8 patients suffered from CRC-related death. Dividing these 20 patients into two groups based on the median preoperative serum AFP level, we found that the AFP-high group had worse progression-free survival (PFS) than the AFP-low group did. However, there was no significant association between the serum AFP level and PFS ( $P=0.578$, Figure $4 \mathrm{~A})$ or overall survival (OS) ( $P=0.885$, Figure 4B). Compared to the 40 staged-matched control patients, the AFP-producing CRC patients exhibited a trend toward worse PFS $(P=0.349$, Figure $4 \mathrm{C})$ and $\mathrm{OS}(P=0.369$, Figure 4D).

\section{Discussion}

The incidence of AFP-producing gastric cancer (GC) has been reported to be $1.3-15 \%{ }^{4,26-29}$ in different countries. However, the incidence in the colorectum is markedly lower than that in stomach. In this study, we analyzed 20 cases of AFP-producing CRC. We found some similar clinical and pathological characteristics demonstrated in AFP-producing CRC and AFP-producing GC, such as: 1) they were more likely occur in men; 2) elevated serum AFP levels; 3) poor differentiation in most cases; 4) advanced local invasion accounted for a large proportion of tumors; 5) a high likelihood of LN metastasis and distant metastasis; and 6) a poor prognosis. These findings suggested that AFP-producing CRC might have a more aggressive biological behavior than traditional CRC (nonAFP-producing CRC). AFP is a remarkable protein in the fetus, and an increase in serum AFP in these tumors indicates the possibility of retro-differentiation to the prenatal state ${ }^{30}$ and the presentation of original biological behaviors by tumor cells, which may result in poor prognosis.

In our cases, we found three types of histological patterns, including COM, MA and HPT, in which COM was the most common type. Therefore, it was difficult for pathologists to recognize AFP-producing CRC depending on the histological features without AFP serum levels. Thus, information on tumor markers in the serum is the key to the diagnosis of this unique type of CRC. In addition, AFP-producing GC may show other histologic patterns, such as the ENT type, yolk sac tumor type,${ }^{30-33}$ medullary type, papillotubular type, ${ }^{34,35}$ 

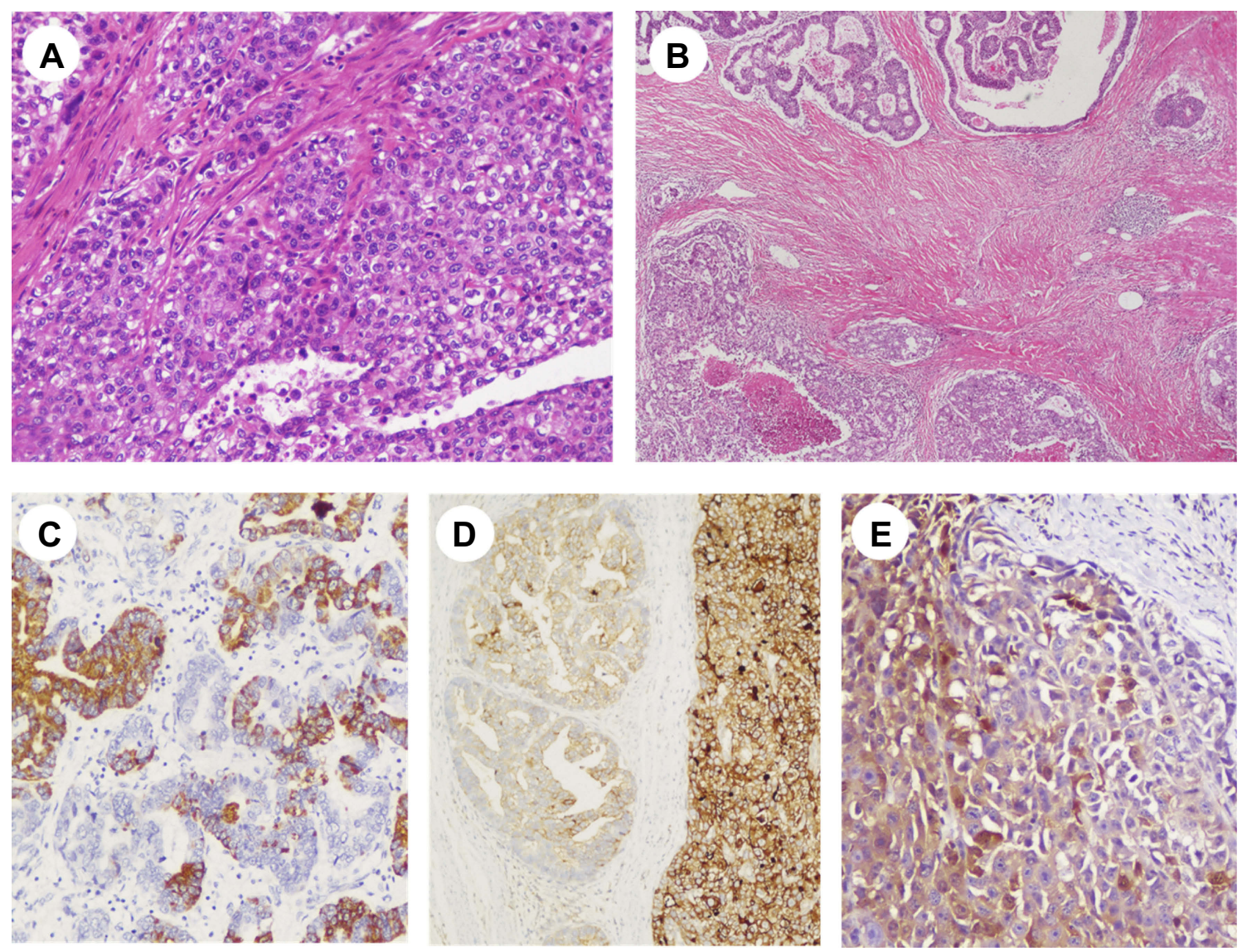

Figure 2 (A) Poorly differentiated CRC composed of hepatoid type (HE, original magnification $\times 200)$. (B) Types of histological transitions in AFP-producing CRC, transition from COM (right side) to HPT (left side) (HE, original magnification×40). (C-E) Immunohistochemical staining: (C) Hepa-I; (D) GPC-3; (E) Arg-I. (immunohistochemistry, original magnification $\times 200$ ).

Abbreviations: COM, common adenocarcinoma type; CRC, colorectal cancer; HPT, hepatoid type.

signet ring cell type and undifferentiated type. ${ }^{29}$ Tumors arising from the stomach seemed to have a more varied histological type than those from the colorectum, but only a limited number of CRC cases were reported. Notably, the HPT was a distinct histological pattern due to the unique IHC expression of GPC3, Hepa-1 and Arg-1, which suggested hepatocellular differentiation. ${ }^{36,37}$ Carcinomas with hepatocellular differentiation are also called hepatoid adenocarcinomas. Most hepatoid adenocarcinomas can be detected by elevated AFP levels in the serum, but in some cases, AFP levels are not increased. AFP-producing cancers are not always derived from hepatoid adenocarcinomas. ${ }^{33}$ It has been reported that $40.1 \%$ of AFP-producing GC cases are hepatoid adenocarcinomas. ${ }^{5}$ Similarly, in our examination of AFP-producing CRCs, only 1 case (5\%) showed a pure HPT pattern, and 5 cases $(25 \%)$ exhibited a partial HPT pattern. Obviously, hepatoid adenocarcinomas are more rare among AFP-producing CRC than AFP-producing GC, which indicates that the mechanisms of hepatoid adenocarcinomas and AFP-producing CRCs are likely different from those of GCs.

There are two main theories about the origin of AFPproducing cancers. Some researchers found that in many cases, AFP-producing GCs develop as the COM type in the mucosa and differentiate into the ENT type and HPT during the process of tumor invasion and proliferation, acquiring the ability to produce AFP. ${ }^{31}$ Fujii et al performed gene analysis and found that $13 \mathrm{qLOH}$ was selectively observed in AFPpositive ENT and HPT in the area of invasion, suggesting that changes in histological morphology and the ability to produce AFP are associated with genetic changes. ${ }^{38}$ Other changes lead to the theory of retrodifferentiation to the fetal gut ${ }^{30}$ or yolk sac tumor. Li et al found that autocrine HGF/c-Met activation may be able to induce dedifferentiation of common adenocarcinoma cells, which revert to a stem cancer cell phenotype and produce AFP or hepatoid differentiation. ${ }^{17}$ Regardless of which theories, all of them support the fact that AFP-producing cancer may arise from traditional 
A

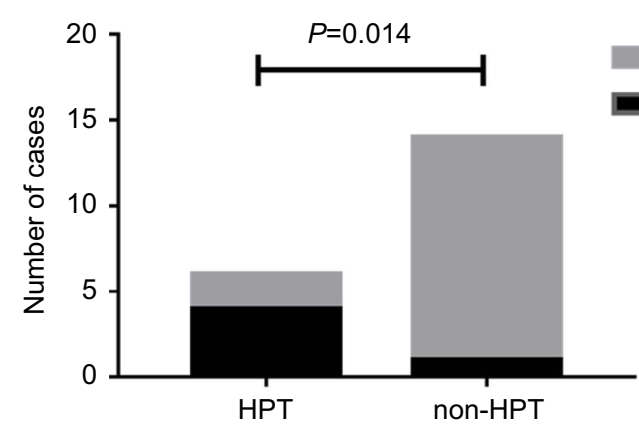

C

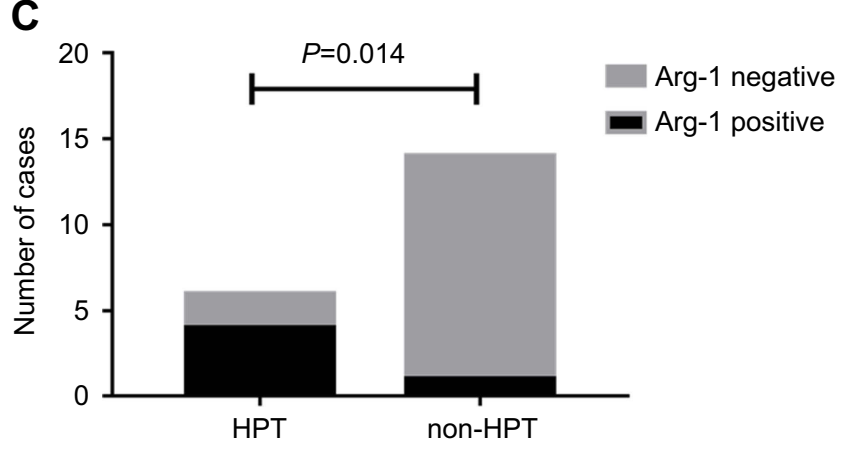

B

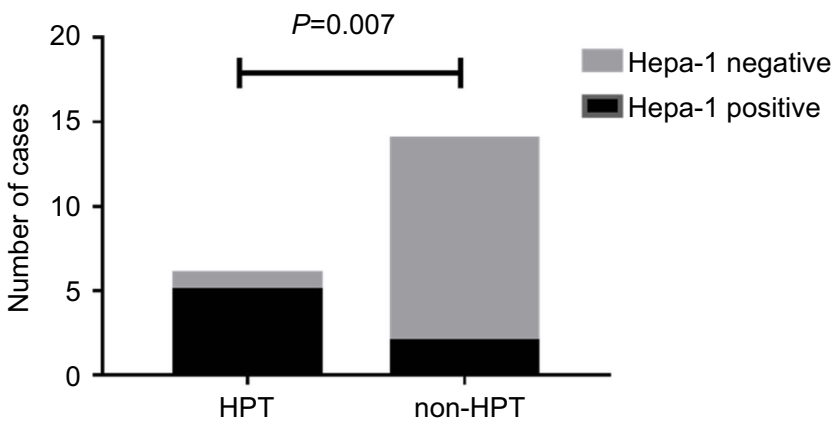

Figure 3 Comparisons of immunohistochemical staining in different histological patterns. The positive staining rate of GPC3 (A), Hepa-I (B) and Arg-I (C) was significantly higher in the HPT than in the non-HPT.

Abbreviation: HPT, hepatoid type.

adenocarcinomas and acquire the ability to produce AFP during progression. Others found that this type of tumor may be promoted by inflammatory bowel disease, which could also lead to traditional adenocarcinomas. ${ }^{21,23-25}$ In our results, one patient had MMR deficiency, and another harbored a KRAS exon 4 mutation, which can also be detected in traditional adenocarcinomas. However, additional studies are needed to prove that AFP-producing cancer and traditional adenocarcinomas share the same carcinogenesis.

Serum AFP is a useful tool to detect AFP-producing cancers. In addition, the level of serum AFP decreased significantly after surgery, which suggests that AFP levels were correlated with tumor load. In our cases, serum AFP was significantly higher in patients with $\mathrm{LN}$ metastasis than in patients without LN metastasis, suggesting its potential value to predict tumor recurrence and for therapy monitoring. We also found a tendency for high serum AFP to be positively related to distant metastasis, advanced TNM stage and poor PFS, although without significance, which was most likely due to the small number of cases. These results were consistent with other investigations. AFP-producing CRC patients with liver metastasis had extremely high serum AFP levels before surgery. ${ }^{13}$ In AFP-producing GC, high serum AFP levels were also significantly correlated with metachronous liver metastasis in a report including 104 patients. ${ }^{27}$ Therefore, serum AFP was useful to identify patients at a high risk of liver metastasis and for whom prophylactic treatment was needed. ${ }^{27}$ The serum AFP level was also reported as a prognostic factor in AFP-producing GC. ${ }^{39}$ In conclusion, serum AFP was an important marker for patients with AFP-producing cancer. It might be used to 1) detect and help diagnose AFP-producing cancer; 2) monitor tumor load and aid in follow-up; 3) indicate residual tumor and liver metastasis; and 4) indicate the prognosis.

The survival analysis demonstrated that patients with AFPproducing CRC had a poorer prognosis than patients with traditional CRC (non-AFP-producing CRC). This finding was confirmed in other case reports of $\mathrm{CRC}^{13,19}$ and investigations of stomach cancer. ${ }^{29,39}$ However, no difference in survival was observed between patients with the HPT pattern and those with other patterns among the 20 cases in our study. According to the literature, hepatoid adenocarcinoma of the stomach, irrespective of AFP production, had a poorer prognosis than AFP-producing GC without hepatoid differentiation. ${ }^{40}$ Thus, we should distinguish hepatoid adenocarcinoma and non-hepatoid differentiated carcinoma among AFP-producing GCs, ${ }^{5}$ for which high levels of serum AFP 

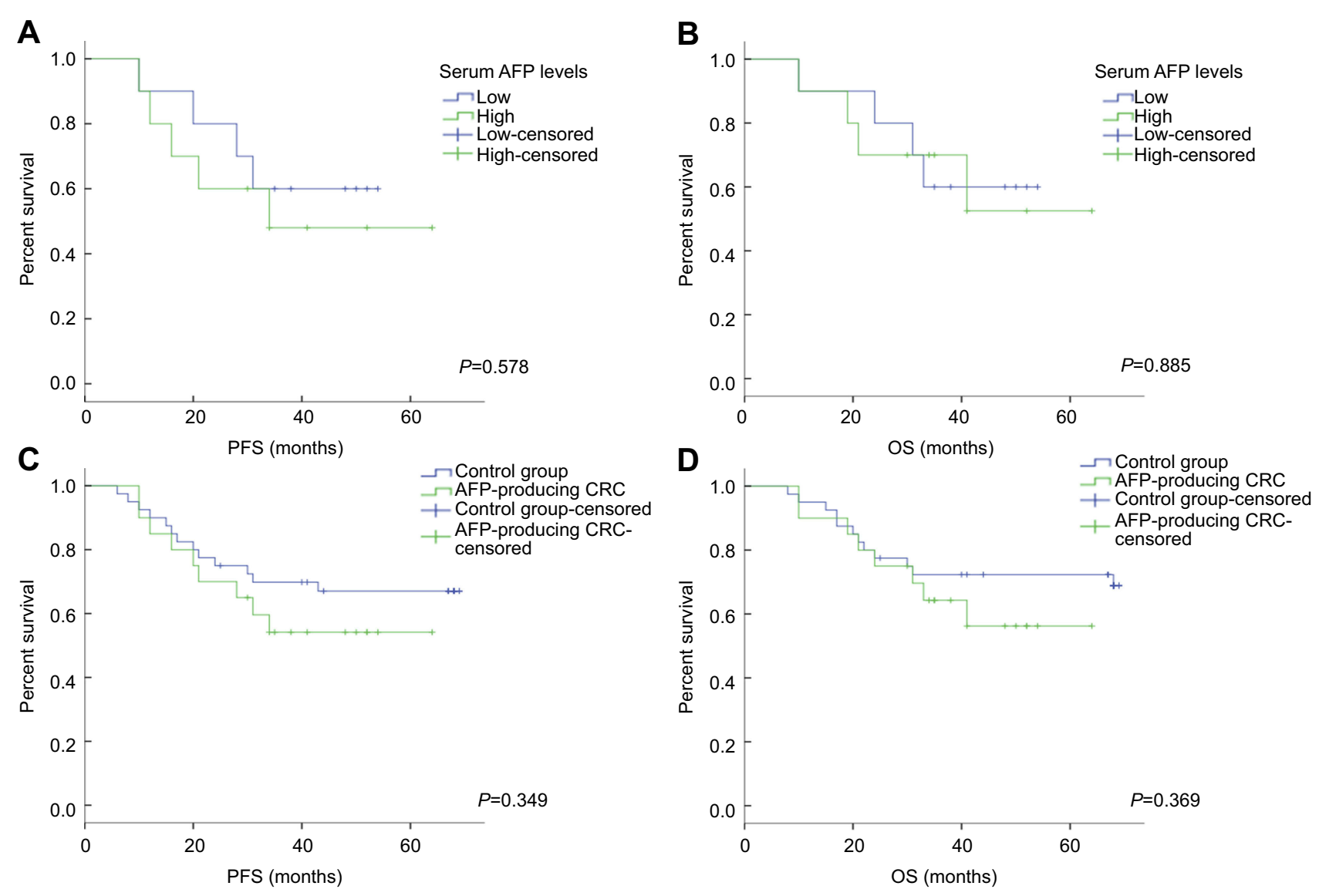

Figure 4 Kaplan-Meier estimates of PFS (A) and OS (B) among patients with low serum AFP levels and high serum AFP levels. Kaplan-Meier estimates of PFS (C) and OS (D) among patients in the AFP-producing CRC and control groups. $(P<0.05)$.

Abbreviations: CRC, colorectal cancer; OS, overall survival; PFS, progression-free survival.

predict a poor outcome. ${ }^{35}$ In our study of CRC, a trend was observed in which the serum AFP-high group had worse PFS than the AFP-low group. More cases of AFP-producing CRC should be investigated to confirm these results.

Treatment of AFP-producing CRC was usually according to traditional adenocarcinomas as resection of the tumor followed by adjuvant chemotherapy if needed. The effect of treatment varied. It is regrettable that some AFP-producing cancers are resistant to multiple drugs. Zeng et al found that PFS and disease-specific survival of intestinal hepatoid adenocarcinoma had no relationship with chemotherapy, which may indicate that this type of tumor is not sensitive to typical chemotherapy agents. $\mathrm{Li}$ et al presented three cases of AFP-producing CRC resistance to multiple drugs, including epidermal growth factor receptor inhibitors. ${ }^{17}$ It was promising that partial patients could benefit from preoperative chemoradiotherapy. ${ }^{16}$ Adjuvant chemotherapy might also help patients. ${ }^{14,41}$ A study of AFP-producing GC found that patients could respond to anti-angiogenic drugs such as ramucirumab. ${ }^{42}$ Percutaneous ethanol injection may be an effective method of treating metastatic liver lesions. ${ }^{27}$ More studies are necessary to determine the most suitable strategies for treating AFP-producing CRC.

\section{Conclusion}

AFP-producing CRC is a rare tumor characterized by an increased possibility of poor differentiation, advanced local invasion, LN metastasis and distant metastasis. The histological patterns of AFP-producing CRC included COM, MA and HPT. Among them, the HPT pattern was the most distinct for high positivity rates of GPC3, Hepa-1 and Arg-1, which suggested hepatocellular differentiation. Serum AFP levels showed potential value in predicting recurrence. AFP-producing CRC had more aggressive biological behaviors and poorer prognosis than did non-AFP-producing CRC. Therefore, special treatment strategies might be needed for this unique tumor type. 


\section{Acknowledgments}

This study was supported by the National Human Genetic Resources Sharing Service Platform (2005DKA21300), National Natural Science Foundation of China (81602078, 81472220, 81802367, 81802361), Shanghai Science and Technology Development Fund (17ZR1406500 and 15ZR 1407400), and Hospital Foundation of Fudan University Shanghai Cancer Center (YJ201704).

\section{Disclosure}

The authors report no conflicts of interest in this work.

\section{References}

1. Bergstrand CG, Czar B. Demonstration of a new protein fraction in serum from the human fetus. Scand J Clin Lab Invest. 1956;8(2):174. doi:10.3109/00365515609049266

2. Smith CJ, Ajdukiewicz A, Kelleher PC. Concanavalin-A-affinity molecular heterogeneity of human hepatoma AFP and cord-serum AFP. Ann N Y Acad Sci. 1983;417:69-74.

3. Norgaard-Pedersen B, Albrechtsen R, Teilum G. Serum alphafoetoprotein as a marker for endodermal sinus tumour (yolk sac tumour) or a vitelline component of "teratocarcinoma". Acta Pathologica et Microbiologica Scand Sec A Pathol. 1975;83 (6):573-589.

4. McIntire KR, Waldmann TA, Moertel CG, Go VL. Serum alpha-fetoprotein in patients with neoplasms of the gastrointestinal tract. Cancer Res. 1975;35(4):991-996.

5. Liu X, Sheng W, Wang Y. An analysis of clinicopathological features and prognosis by comparing hepatoid adenocarcinoma of the stomach with AFP-producing gastric cancer. J Surg Oncol. 2012;106 (3):299-303. doi:10.1002/jso.23073

6. Nakajima T, Okazaki N, Morinaga S, Tsumuraya M, Shimosato Y, Saiki S. A case of alpha-fetoprotein-producing rectal carcinoma. Jpn $J$ Clin Oncol. 1985;15(4):679-685.

7. Yu YY, Ogino T, Okada S. An alpha-fetoprotein-producing carcinoma of the rectum. Acta Pathol Jpn. 1992;42(9):684-687.

8. Sato Y, Sekine T, Ohwada S. Alpha-fetoprotein-producing rectal cancer: calculated tumor marker doubling time. J Surg Oncol. 1994;55 (4):265-268.

9. Hocking GR, Shembrey M, Hay D, Ostor AG. Alpha-fetoproteinproducing adenocarcinoma of the sigmoid colon with possible hepatoid differentiation. Pathology. 1995;27(3):277-279.

10. Kato K, Matsuda M, Ingu A, et al. Colon cancer with a high serum alpha-fetoprotein level. Am J Gastroenterol. 1996;91(5):1045-1046.

11. Taguchi J, Yano H, Sueda J, et al. alpha-Fetoprotein-producing rectal carcinoma-a case report. Kurume Med J. 1997;44(4):339-348.

12. Kurihara K, Konishi F, Kanazawa K, Fujii T, Saito K. Alphafetoprotein-producing carcinoma of the colon: report of a case. Surg Today. 1997;27(5):453-456.

13. Yachida S, Fukushima N, Nakanishi Y, et al. Alpha-fetoproteinproducing carcinoma of the colon: report of a case and review of the literature. Dis Colon Rectum. 2003;46(6):826-831. doi:10.1097/ 01.DCR.0000070038.81534.F7

14. Fu K, Kobayashi A, Saito N, et al. Alpha-fetoprotein-producing colon cancer with atypical bulky lymph node metastasis. World J Gastroenterol. 2006;12(47):7715-7716.

15. Anzai H, Kazama S, Kiyomatsu T, et al. Alpha-fetoprotein-producing early rectal carcinoma: a rare case report and review. World J Surg Oncol. 2015;13:180. doi:10.1186/s12957-015-0590-x
16. Nakamura Y, Matsuda K, Yokoyama S, et al. Alpha-fetoproteinproducing rectal cancer successfully responded to preoperative chemoradiotherapy: case report. Surg Case Rep. 2018;4(1):111. doi:10.1186/s40792-018-0520-6

17. Li J, Liu Y, Xu JH, Xu ZP, Zheng S, Ding KF. Expression of hepatocyte growth factor and c-Met is characteristic of alpha-fetoprotein-producing colorectal adenocarcinoma: a report of three cases. Oncol Lett. 2016;11 (1):731-734. doi:10.3892/ol.2015.3964

18. Valentino F, Torchio M, Morbini P, Danova M. Synchronous presentation of hepatoid alpha-fetoprotein-producing lung cancer and colorectal adenocarcinoma. Tumori. 2012;98(5):130e-134e. doi:10.1700/ 1190.13214

19. Cappetta A, Bergamo F, Mescoli C, Lonardi S, Rugge M, Zagonel V. Hepatoid adenocarcinoma of the colon: what should we target? Pathol Oncol Res. 2012;18(1):93-96. doi:10.1007/s12253-0119424-5

20. Armaghani A, Hernandez Gonzalo D, Daily K. Hepatoid adenocarcinoma of the colon. BMJ Case Rep. 2015;2015.

21. Chen Y, Schaeffer DF, Yoshida EM. Hepatoid adenocarcinoma of the colon in a patient with inflammatory bowel disease. World $J$ Gastroenterol. 2014;20(35):12657-12661. doi:10.3748/wjg.v20.i35.12657

22. Ishikura $H$, Kishimoto $T$, Andachi $H$, Kakuta $Y$, Yoshiki $T$. Gastrointestinal hepatoid adenocarcinoma: venous permeation and mimicry of hepatocellular carcinoma, a report of four cases. Histopathology. 1997;31(1):47-54.

23. Lattes C, Carella R, Faggioli S, Gabusi E, Grigioni WF. Hepatoid adenocarcinoma of the rectum arising in ulcerative colitis: report of a case. Dis Colon Rectum. 2000;43(1):105-108.

24. Borgonovo G, Razzetta F, Assalino M, Varaldo E, Puglisi M, Ceppa P. Rectal hepatoid carcinoma with liver metastases in a patient affected by ulcerative colitis. Hepatobiliary Pancreat Dis Int. 2008;7(5):539-543.

25. Zeng X, Zhang P, Xiao H, et al. Clinicopathological features and prognosis of intestinal hepatoid adenocarcinoma: evaluation of a pooled case series. Oncotarget. 2018;9(2):2715-2725. doi:10.18632/oncotarget.23595

26. Kono K, Amemiya H, Sekikawa T, et al. Clinicopathologic features of gastric cancers producing alpha-fetoprotein. Dig Surg. 2002;19 (5):359-365; discussion 365. doi:10.1159/000065838

27. Liu X, Cheng Y, Sheng W, et al. Clinicopathologic features and prognostic factors in alpha-fetoprotein-producing gastric cancers: analysis of 104 cases. J Surg Oncol. 2010;102(3):249-255. doi:10.1002/jso. 21624

28. Sun W, Liu B, Chen J, et al. Novel characteristics of alpha-fetoprotein (AFP)-producing gastric cancer. Oncotarget. 2017;8(60):101944-101951. doi:10.18632/oncotarget.22109

29. $\mathrm{Li} \mathrm{XD}, \mathrm{Wu} \mathrm{CP}, \mathrm{Ji} \mathrm{M}$, et al. Characteristic analysis of alpha-fetoprotein-producing gastric carcinoma in China. World J Surg Oncol. 2013;11:246. doi:10.1186/1477-7819-11-246

30. Matsunou H, Konishi F, Jalal RE, Yamamichi N, Mukawa A. Alpha-fetoprotein-producing gastric carcinoma with enteroblastic differentiation. Cancer. 1994;73(3):534-540.

31. Kinjo T, Taniguchi H, Kushima R, et al. Histologic and immunohistochemical analyses of alpha-fetoprotein-producing cancer of the stomach. Am J Surg Pathol. 2012;36(1):56-65. doi:10.1097/ PAS.0b013e31823aafec

32. Motoyama T, Aizawa K, Watanabe H, Fukase M, Saito K. Alphafetoprotein producing gastric carcinomas: a comparative study of three different subtypes. Acta Pathol Jpn. 1993;43(11):654-661.

33. Ooi A, Nakanishi I, Sakamoto N, et al. Alpha-fetoprotein (AFP)-producing gastric carcinoma. Is it hepatoid differentiation? Cancer. 1990;65(8):1741-1747.

34. Kodama T, Kameya T, Hirota T, et al. Production of alpha-fetoprotein, normal serum proteins, and human chorionic gonadotropin in stomach cancer: histologic and immunohistochemical analyses of 35 cases. Cancer. 1981;48(7):1647-1655. 
35. Uefuji K, Ichikura T, Tamakuma S. Roles of histological findings and serum AFP levels in the prognosis of AFP-producing gastric cancers. Jpn J Clin Oncol. 1994;24(3):135-140.

36. Villari D, Caruso R, Grosso M, Vitarelli E, Righi M, Barresi G. Hep Par 1 in gastric and bowel carcinomas: an immunohistochemical study. Pathology. 2002;34(5):423-426.

37. McKnight R, Nassar A, Cohen C, Siddiqui MT. Arginase-1: a novel immunohistochemical marker of hepatocellular differentiation in fine needle aspiration cytology. Cancer Cytopathol. 2012;120(4):223-229. doi:10.1002/cncy.21184

38. Fujii H, Ichikawa K, Takagaki T, et al. Genetic evolution of alpha fetoprotein producing gastric cancer. J Clin Pathol. 2003;56 (12):942-949.
39. He R, Yang Q, Dong X, et al. Clinicopathologic and prognostic characteristics of alpha-fetoprotein-producing gastric cancer. Oncotarget. 2017;8(14):23817-23830. doi:10.18632/oncotarget.15909

40. Nagai E, Ueyama T, Yao T, Tsuneyoshi M. Hepatoid adenocarcinoma of the stomach. A clinicopathologic and immunohistochemical analysis. Cancer. 1993;72(6):1827-1835.

41. Slotta JE, Jungling B, Kim YJ, Wagner M, Igna D, Schilling MK. Hepatoid adenocarcinoma of the transverse colon. Int $J$ Colorectal Dis. 2012;27(7):989-991. doi:10.1007/s00384-011-1305-6

42. Arakawa Y, Tamura M, Aiba K, et al. Significant response to ramucirumab monotherapy in chemotherapy-resistant recurrent alpha-fetoprotein-producing gastric cancer: a case report. Oncol Lett. 2017;14(3):3039-3042. doi:10.3892/ol.2017.6514 


\section{Supplementary material}

Table SI Clinicopathological characteristics of 40 stage-matched patients with traditional CRC (non-AFP-producing CRC)

\begin{tabular}{|l|l|}
\hline Characteristics & N (\%) \\
\hline Age (years) & \\
$\leq 60$ & $22(55 \%)$ \\
$>60$ & $18(45 \%)$ \\
\hline Sex & \\
Male & $25(63 \%)$ \\
Female & $15(38 \%)$ \\
\hline Location & \\
Ascending & $8(20 \%)$ \\
Transverse & $3(8 \%)$ \\
Descending & $1(3 \%)$ \\
Sigmoid & $16(40 \%)$ \\
Rectum & $12(30 \%)$ \\
\hline Differentiation & \\
Poorly differentiated & $16(40 \%)$ \\
Moderately differentiated & $23(58 \%)$ \\
Well differentiated & $1(3 \%)$ \\
\hline pTNM stage & \\
I & $2(5 \%)$ \\
II & $8(20 \%)$ \\
III & $16(40 \%)$ \\
IV & $14(35 \%)$ \\
\hline
\end{tabular}

Notes: ${ }^{\text {a }}$ patients were staged in accordance with the 8 th edition of the AJCC TNM classification.

Abbreviations: AJCC, American Joint Committee on Cancer; CRC, colorectal cancer.

\section{Publish your work in this journal}

Cancer Management and Research is an international, peer-reviewed open access journal focusing on cancer research and the optimal use of preventative and integrated treatment interventions to achieve improved outcomes, enhanced survival and quality of life for the cancer patient.
The manuscript management system is completely online and includes a very quick and fair peer-review system, which is all easy to use. Visit http://www.dovepress.com/testimonials.php to read real quotes from published authors. 